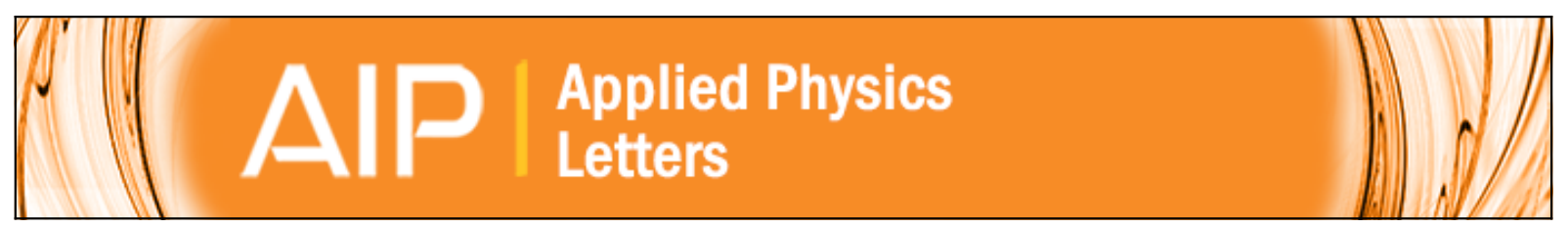

\title{
Nano-porosity in GaSb induced by swift heavy ion irradiation
}

P. Kluth, J. Sullivan, W. Li, R. Weed, C. S. Schnohr, R. Giulian, L. L. Araujo, W. Lei, M. D. Rodriguez, B. Afra, T. Bierschenk, R. C. Ewing, and M. C. Ridgway

Citation: Applied Physics Letters 104, 023105 (2014); doi: 10.1063/1.4861747

View online: http://dx.doi.org/10.1063/1.4861747

View Table of Contents: http://scitation.aip.org/content/aip/journal/apl/104/2?ver=pdfcov

Published by the AIP Publishing

\section{Articles you may be interested in}

Argon-ion-induced formation of nanoporous GaSb layer: Microstructure, infrared luminescence, and vibrational properties

J. Appl. Phys. 116, 033514 (2014); 10.1063/1.4890608

Nanoporosity induced by ion implantation in deposited amorphous Ge thin films

J. Appl. Phys. 111, 113515 (2012); 10.1063/1.4725427

Porosity-induced relaxation of strains in GaN layers studied by means of micro-indentation and optical spectroscopy

J. Appl. Phys. 111, 093513 (2012); 10.1063/1.4710994

Thermal evolution of microstructure in ion-irradiated GaN

J. Appl. Phys. 105, 083514 (2009); 10.1063/1.3106606

Ion-irradiation-induced porosity in GaSb

Appl. Phys. Lett. 86, 131920 (2005); 10.1063/1.1896428

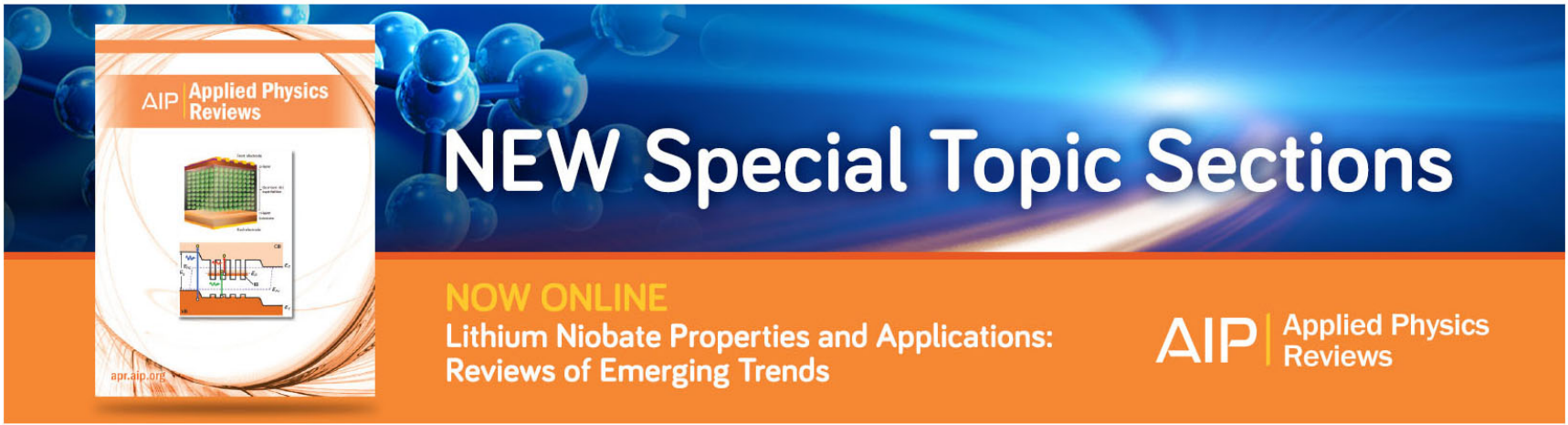




\title{
Nano-porosity in GaSb induced by swift heavy ion irradiation
}

\author{
P. Kluth, ${ }^{1, \text { a) }}$ J. Sullivan, ${ }^{2}$ W. Li, ${ }^{3}$ R. Weed, ${ }^{2}$ C. S. Schnohr, ${ }^{1, b)}$ R. Giulian, ${ }^{1, c)}$ L. L. Araujo, ${ }^{1, c)}$ \\ W. Lei, ${ }^{1, d)}$ M. D. Rodriguez, ${ }^{1}$ B. Afra,${ }^{1}$ T. Bierschenk, ${ }^{1}$ R. C. Ewing,${ }^{3, e)}$ and M. C. Ridgway ${ }^{1}$ \\ ${ }^{1}$ Department of Electronic Materials Engineering, Research School of Physics and Engineering, \\ Australian National University, Canberra, Australian Capital Territory 0200, Australia \\ ${ }^{2}$ ARC Centre for Antimatter-Matter Studies, AMPL, Research School of Physics and Engineering, \\ Australian National University, Canberra, Australian Capital Territory 0200, Australia \\ ${ }^{3}$ Department of Earth and Environmental Sciences, University of Michigan, Ann Arbor, \\ Michigan 48109-1005, USA
}

(Received 28 October 2013; accepted 27 December 2013; published online 13 January 2014)

\begin{abstract}
Nano-porous structures form in GaSb after ion irradiation with $185 \mathrm{MeV}$ Au ions. The porous layer formation is governed by the dominant electronic energy loss at this energy regime. The porous layer morphology differs significantly from that previously reported for low-energy, ion-irradiated $\mathrm{GaSb}$. Prior to the onset of porosity, positron annihilation lifetime spectroscopy indicates the formation of small vacancy clusters in single ion impacts, while transmission electron microscopy reveals fragmentation of the GaSb into nanocrystallites embedded in an amorphous matrix. Following this fragmentation process, macroscopic porosity forms, presumably within the amorphous phase. (C) 2014 AIP Publishing LLC. [http://dx.doi.org/10.1063/1.4861747]
\end{abstract}

Antimony based semiconductors, such as GaSb and InSb, have many important applications, including laser diodes, high frequency electronic devices, ${ }^{1}$ high efficiency infra-red photodetectors, as well as high-efficiency thermophotovoltaic systems and tandem concentrator solar cells. ${ }^{2,3}$ Nano-porous semiconductors have been identified as ideal building blocks for optoelectronic, photovoltaic and sensor devices, and membranes for biological and chemical applications. ${ }^{4,5}$ A commonly used method for fabricating porous semiconductors is electrochemical etching. This has been extensively studied for the fabrication of porous silicon, ${ }^{6}$ but has also been reported for compound semiconductors, such as $\mathrm{GaAs}$, InP, and $\mathrm{GaP}^{7}$ In contrast to electrochemical etching methods, using ion irradiation to induce porosity is largely independent of the electrical properties of the material and provides a technology that is compatible with industrial device fabrication; however, few semiconductor materials can be rendered porous using this technique. In the low energy regime, where nuclear stopping is predominant, the formation of porous structures has been reported for $\mathrm{Ge}$, $\mathrm{GaSb}$, and InSb. ${ }^{8-16}$ In general, the formation of porosity is attributed to clustering of vacancies that are generated during the elastic collisions when the material is irradiated by energetic ions. In the case of Ge, porosity was only observed after the material was first rendered amorphous. ${ }^{16}$ The current understanding is that the amorphous structure can accommodate more interstitial-like atoms with the consequence that vacancy clustering is enhanced. In contrast to $\mathrm{Ge}$, the

\footnotetext{
a) Author to whom correspondence should be addressed. Electronic mail: patrick.kluth@anu.edu.au

b)Present address: Institut für Festkörperphysik, Friedrich-SchillerUniversität Jena, Max-Wien-Platz 1, 07743 Jena, Germany.

${ }^{c}$ Present address: Instituto de Fisica, Universidade Federal do Rio Grande do Sul, Porto Alegre, Brazil.

${ }^{d)}$ Present address: School of Electrical, Electronic and Computer Engineering, The University of Western Australia, Crawley, Western Australia 6009, Australia.

${ }^{\text {e) }}$ Present address: Geological and Environmental Sciences, Stanford University, Stanford, California 94305, USA.
}

continuous-to-porous transformation in $\mathrm{GaSb}$ and $\mathrm{InSb}$ occurs simultaneously with the crystalline-to-amorphous transformation and is attributed to inefficient Frenkel pair (i.e., interstitial and vacancy pair) recombination. ${ }^{10,16}$ It remains unclear why other semiconductors, such as $\mathrm{Si}$, are not rendered porous by low energy ion irradiation.

Using high-energy irradiation, which is dominated by electronic energy loss, recent reports show porous structure formation in Ge. ${ }^{17,18}$ As in the case of low energy irradiation, only amorphous Ge becomes porous. In this investigation, we present results on the formation of nano-porous structures in GaSb using swift heavy ion irradiation. The resulting structures are distinctly different from those formed by low energy irradiation and the process does not require the material to be amorphous before porosity develops.

For our experiments, we utilized bulk GaSb substrates, as well as $2.2 \mu \mathrm{m}$ thick GaSb layers grown by metal organic chemical vapor deposition (MOCVD) onto InP substrates. Irradiation was performed at room temperature with $185 \mathrm{MeV}{ }^{197} \mathrm{Au}$ ions at the Australian National University Heavy Ion Accelerator Facility with the ion beam normal to the sample surface. Fluences ranged between $1 \times 10^{11}$ and $2 \times 10^{14}$ ions $/ \mathrm{cm}^{2}$. The ion flux was below $5 \times 10^{10}$ ions $/ \mathrm{cm}^{2} / \mathrm{s}$ for all irradiation experiments resulting in power densities below $1.5 \mathrm{~W} / \mathrm{cm}^{2}$. We thus rule out ion beam-induced sample heating. Samples were studied using scanning electron microscopy (SEM), positron annihilation lifetime spectroscopy (PALS), transmission electron microscopy (TEM), as well as small-angle X-ray scattering (SAXS). For SEM analysis, the samples were cleaved and imaged in cross-section. PALS was performed at the Australian Positron Beamline Facility at the ANU using $6 \mathrm{keV}$ positrons. ${ }^{19}$ The penetration depth of the positrons peaks is at approximately $100 \mathrm{~nm}$ for this energy. In order to measure the lifetime spectra, a $\mathrm{BaF}_{2}$ scintillator was used in conjunction with a photo multiplier tube (PMT) and digital multichannel analyzer with $100 \mathrm{ps}$ bin-width. A total of $\sim 1.5 \times 10^{6}$ counts were measured in each spectrum, with a 
typical positron pulse Full-Width Half-Maximum (FWHM) of $\sim 0.8 \mathrm{~ns}$ and signal-to-background ratio of $>10^{5}$. An un-irradiated GaSb sample was used as a reference for the positron measurements, and lifetimes measured in the irradiated samples can then be attributed solely to the damage induced. For TEM studies, the irradiated specimens were thinned to a thickness of $\sim 10 \mu \mathrm{m}$ by polishing the unirradiated surface, followed by continued thinning from the same side by ion milling with a few $\mathrm{keV}$ Ar ions at less than $5^{\circ}$ incidence until perforation. TEM was conducted using a JEOL 3011 electron microscope. Transmission SAXS measurements were performed at the SAXS/WAXS beamline at the Australian Synchrotron. Thin film GaSb samples were utilized where the InP substrate was locally removed by selective wet chemical etching prior to ion irradiation.

Fig. 1(a) shows a cross-sectional SEM image of bulk GaSb irradiated to a fluence of $4 \times 10^{13}$ ions $/ \mathrm{cm}^{2}$. Three distinct regions can be observed. The panels at the top of Fig. 1(a) show magnifications of these regions. In the first region (region 1) that extends to a depth of $\sim 9 \mu \mathrm{m}$, large elongated voids $(>100 \mathrm{~nm})$ are apparent. Beyond this depth, the voids vanish, and the material appears largely solid (region 2). At a depth of about $12 \mu \mathrm{m}$, a layer of small, largely spherical voids with diameters around $10 \mathrm{~nm}$ appears

(a)

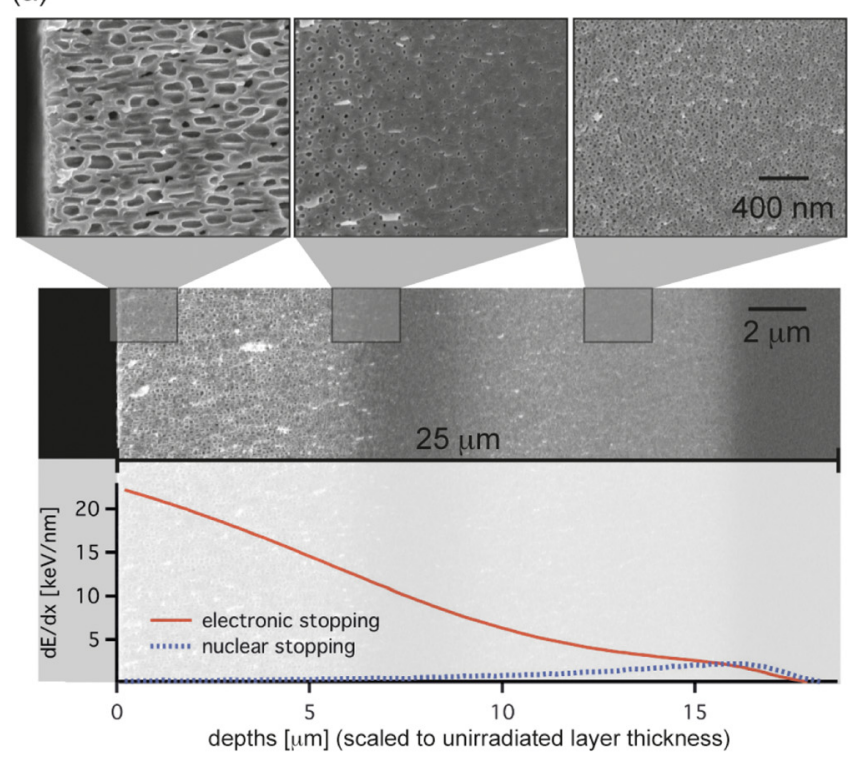

(b)

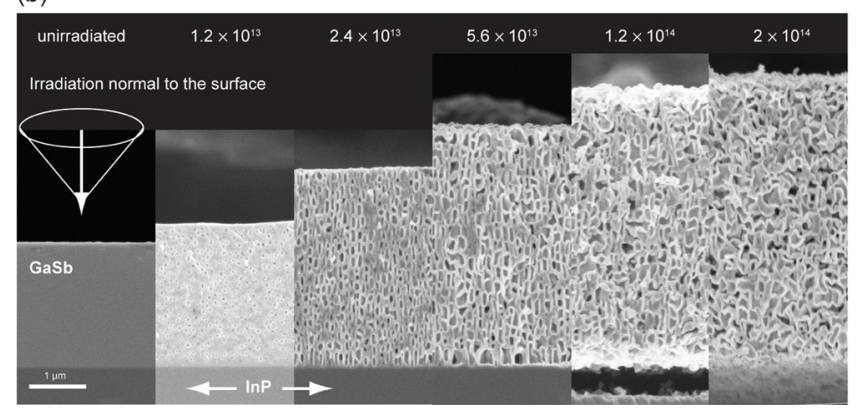

FIG. 1. Cross sectional SEM images of GaSb layers irradiated with $185 \mathrm{MeV}$ Au ions: (a) bulk sample exposed to a fluence of $4 \times 10^{13}$ ions $/ \mathrm{cm}^{2}$. The top panels show magnifications from three characteristic areas. The main image is overlaid with a SRIM calculation of the energy loss as a function of depth, stretched by the swelling of the layer measured by profilometry; (b) GaSb thin films grown on $\mathrm{InP}$ as a function of the irradiation fluence. that extends to a depth of $\sim 22 \mu \mathrm{m}$ (region 3 ). The porosity is accompanied by a swelling of the surface of approximately $6 \mu \mathrm{m}$ that was measured by surface profilometry. At the bottom of the image, a Stopping and Range of Ions in Matter $(\text { SRIM2008) })^{20}$ calculation of the electronic and nuclear energy loss as a function of depth is shown. As the calculation does not account for swelling, it was stretched to match the swollen GaSb layer of $25 \mu$ m layer with an initial thickness of approximately $19 \mu \mathrm{m}$. We are aware that the swelling is not linear over the depth under investigation, and thus, note that the "stretched" SRIM calculation can only be seen as a crude approximation. It is readily apparent, however, that the large voids in region 1 result from the electronic energy deposition, as nuclear stopping is negligible in this area. When the electronic energy loss falls below a value of approximately $10 \mathrm{keV} / \mathrm{nm}$, the voids vanish and the material is continuous (region 2). This value thus provides a threshold for the electronic energy loss at which porosity occurs. The appearance of small spherical voids in region 3 can be attributed to nuclear stopping and is consistent with previous reports. ${ }^{9,10,15}$ In order to isolate the effects of electronic stopping, the porosity was studied in $2.2 \mu \mathrm{m}$ thick GaSb layers grown on InP by MOCVD. The InP substrate remains continuous upon swift heavy ion irradiation. The evolution of the porosity as a function of fluence is shown in Fig. 1(b). The porosity proceeds by the formation of spherical voids, which grow with increasing irradiation fluence into elongated voids and finally irregular pockets with characteristic sizes of $\sim 200 \mathrm{~nm}$ and $\sim 20-30 \mathrm{~nm}$ thick sidewalls. While at low irradiation energies with predominant nuclear stopping, the initial stages of porosity are also characterized by the formation of spherical voids, ${ }^{10}$ the final porous structures resemble open fibrous networks distinctly different from the structures observed here. ${ }^{10,14}$

Using profilometry, the swelling of the material was measured as a function of the ion fluence for both bulk samples, as well as thin films. The results are shown in Fig. 2(a). Clearly, there is a linear increase in the step-height for the bulk samples. A linear fit to the data indicates a threshold fluence of approximately $9 \times 10^{12}$ ions $/ \mathrm{cm}^{2}$ for the swelling to be measurable. In contrast, swelling of the thin film saturates at approximately $3 \mu \mathrm{m}$. The difference in swelling behavior is a consequence of the limited supply of $\mathrm{GaSb}$ in the thin film. The morphology of the porous structure was found to be identical for both bulk and thin film targets (see Figs. 1(a) and 1(b), for example). More importantly, extrapolation of the swelling for the thin film samples yields an almost identical threshold value for formation of pores in the bulk. While no conclusive data have been reported for the damage cross-sections of swift heavy ion tracks in $\mathrm{GaSb},{ }^{21}$ assuming a typical track diameter of $10 \mathrm{~nm}$, a simple overlap model ${ }^{22}$ yields a fluence of approximately $1 \times 10^{13}$ ions $/ \mathrm{cm}^{2}$ to cover the whole irradiated area, in good agreement with the observed threshold fluence. Thus, pre-damage of the material is required before significant porosity forms.

The sub-threshold fluence region between $1 \times 10^{11}$ and $1 \times 10^{13}$ ions $/ \mathrm{cm}^{2}$ was investigated using PALS. The bulk $\mathrm{GaSb}$ samples were used for these measurements. A single lifetime was observed in each sample and is shown as a function of the ion fluence in Fig. 2(b). An apparent increase in 
the lifetime up to a fluence of $1 \times 10^{13}$ ions $/ \mathrm{cm}^{2}$ can be attributed to the formation of vacancy clusters with increasing size. Comparison with theoretical work on silicon suggests that the smallest lifetime of $\sim 450$ ps corresponds to a vacancy cluster $>\mathrm{V} 10 .{ }^{23,24}$ A simple extrapolation of this model suggests that the size increases to a vacancy cluster of around V40 at the longest lifetime, corresponding to a spherical void of around $1 \mathrm{~nm}$ in diameter. This clearly demonstrates that small vacancy clusters already form in single ion impacts. Growing vacancy clusters in a fluence range where largely isolated ion tracks are formed (i.e., $1 \times 10^{11}$ to $1 \times 10^{12}$ ions $/ \mathrm{cm}^{2}$ ) suggests the diffusion of the vacancies beyond the typical track dimensions of $\sim 10 \mathrm{~nm}$.

In order to study the nature of the damage prior to and after the onset of porosity, we performed high-resolution TEM of bulk GaSb samples irradiated with $3 \times 10^{11}$ and $3 \times 10^{13}$ ions $/ \mathrm{cm}^{2}$, respectively. Figs. 3(a) and 3(b) show images of the former and (c) and (d) of the latter. A fluence of $3 \times 10^{11}$ ions $/ \mathrm{cm}^{2}$ is below the threshold for observable porosity. In agreement with the absence of swelling, no voids could be detected by TEM, yet significant fragmentation of the originally single-crystal structure is apparent. We note that the vacancy clusters detected by PALS are too small for detection by TEM. The morphology resembles randomly
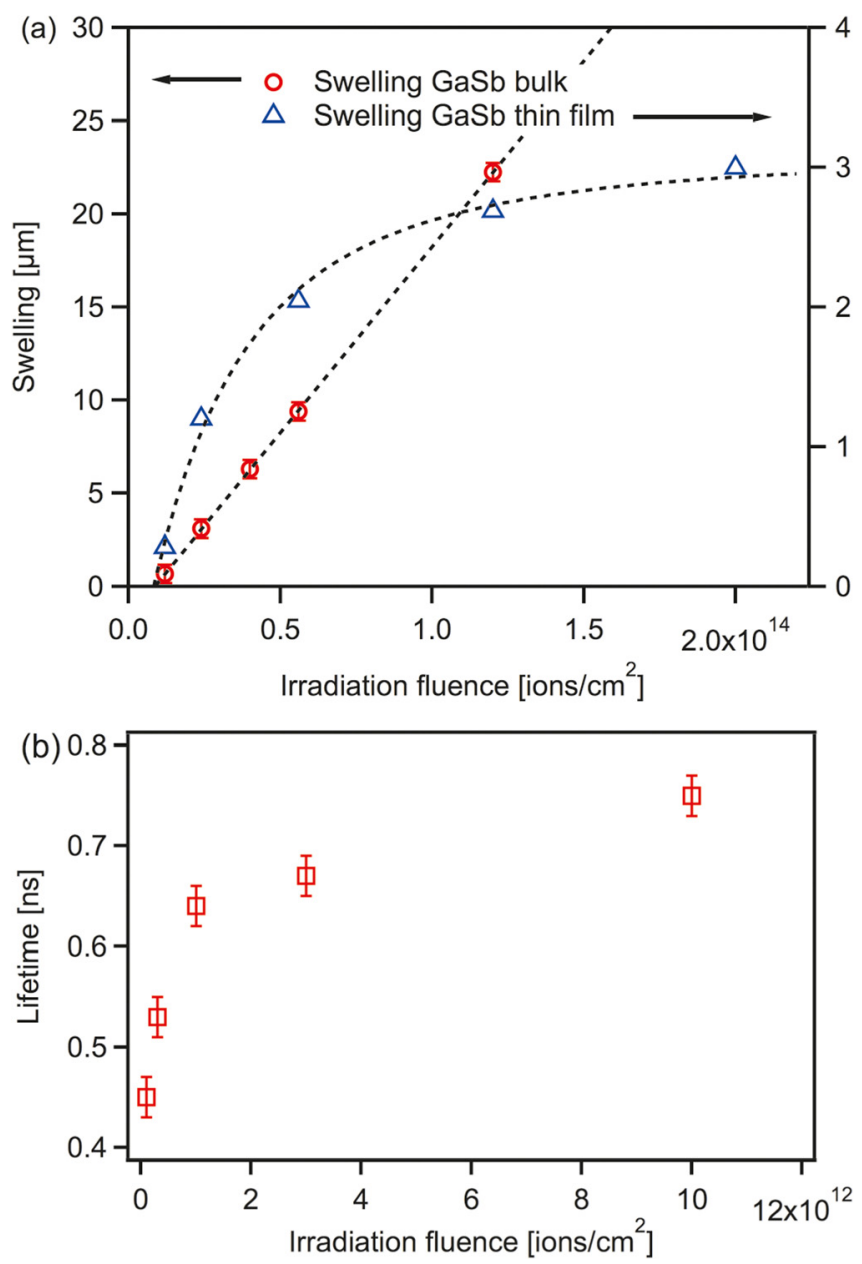

FIG. 2. (a) Swelling of bulk (blue triangles) and thin film (red circles) GaSb measured by profilometry as a function of the irradiation fluence; (b) positron lifetime broadening as a function of the irradiation fluence in the sub-threshold region, i.e., before macroscopic porosity occurs. oriented crystallites, $5-20 \mathrm{~nm}$ in size, embedded in a matrix consisting of amorphous material with "sidewalls" (i.e., the spacing between the crystallites) of a few $\mathrm{nm}$. At a fluence of $3 \times 10^{13}$ ions $/ \mathrm{cm}^{2}$, Fig. 3(c) shows the hollow pockets of the porous structure. A high-resolution image of the solid area in (d) reveals that even at this fluence, where the material experienced multiple ion impacts and porosity is formed, the microstructure still consists of nanocrystallites interspersed in amorphous material. Thus, the formation of porosity during swift heavy ion irradiation does not require the material to be completely amorphous, in contrast to the case of Ge. Electron diffraction analysis shown in the inset of Fig. 3(c) confirms that the crystallites remain in the cubic zincblende structure observed for bulk GaSb and no radiation-induced decomposition was observed.

These results provide important mechanistic insights into the main processes operational during porous layer formation. From the PALS measurements, it is evident that vacancy clusters form within individual ion impacts, however, significant porosity requires a degree of disorder to be present manifested in the observed threshold fluence. Clearly, disorder in the form of dislocations is not the origin of macroscopic porosity, as both the thin layer and bulk GaSb show a very similar evolution of porosity. The former contains a significant dislocation density due to the large lattice mismatch with the InP substrate. Thus, we propose that it is within the amorphous material that the porosity is predominantly generated. In the low energy irradiation case, ${ }^{10,15}$ in contrast, porosity was explained by Ga interstitial agglomeration at extended defects that leave a vacancy excess, which ultimately leads to void formation. Porosity originating in the amorphous phase has been investigated for Ge in both high- and low-energy ion regimes. In the low-energy regime, the amorphous phase can accommodate sufficient interstitials such that enhanced clustering of vacancies can occur, and
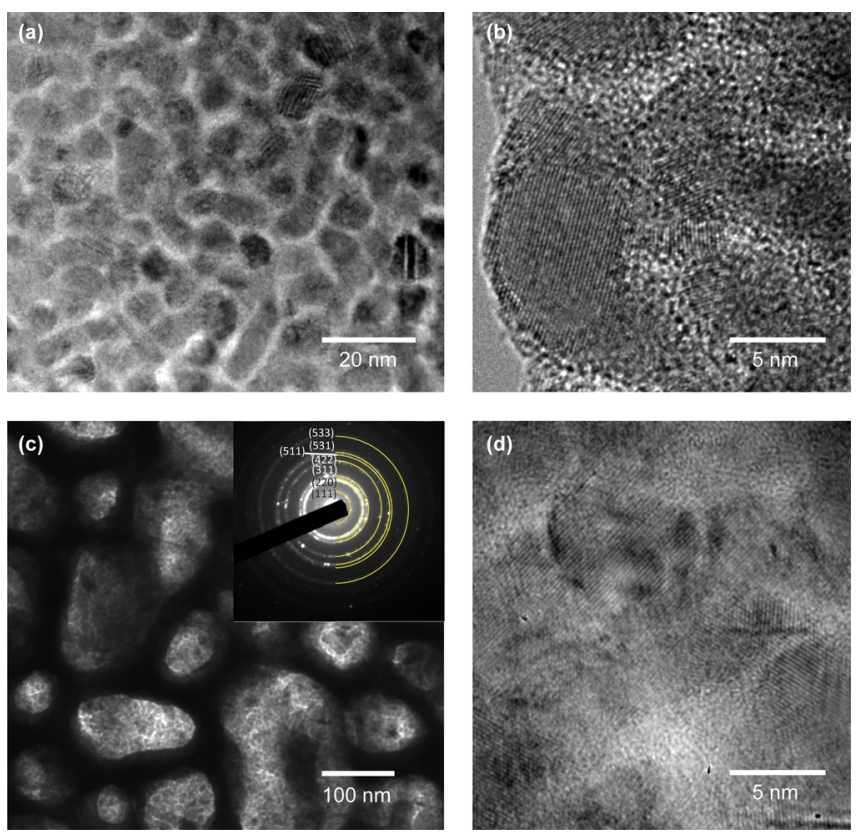

FIG. 3. TEM images of bulk GaSb irradiated with $3 \times 10^{11}$ (a) and (b), and $3 \times 10^{13}$ ions $/ \mathrm{cm}^{2}$ (c) and (d). The inset in (c) shows the selected area electron diffraction pattern. The indexing is consistent with crystallites in different orientations that have the zincblende structure. 
this ultimately leads to macroscopic porosity. ${ }^{8}$ In the highenergy regime, a melt-quench process leads to the formation of macroscopic voids within single ion tracks. ${ }^{25}$ The latter were accompanied by ion tracks with a low atomic-density core and a high atomic-density shell. The TEM observation of randomly oriented nanocrystallites in low fluence irradiated single crystal $\mathrm{GaSb}$ indicates a melt-quench process is also operational during single ion impacts, albeit recrystallisation of part of the material occurs during the quenching process. In contrast to the case of $\mathrm{Ge},{ }^{25}$ transmission SAXS measurements at the Australian Synchrotron on a thin film GaSb sample irradiated with $1 \times 10^{11}$ ions $/ \mathrm{cm}^{2}$ are indistinguishable from those of an unirradiated sample, thus leaving no evidence of ion track or void formation. Such measurements have previously enabled us to determine the track structure in a variety of other materials with great success. $^{22,26-28}$ This suggests that ion tracks in GaSb may be dissimilar to those in other materials that often show amorphous high-aspect ratio track cores in a crystalline matrix. In the current case, resolidification includes recrystallization albeit not epitaxial, leading to the observed fragmentation. The current results cannot unambiguously determine if the void formation in the amorphous part of the GaSb is similar to that in amorphous $\mathrm{Ge}$, as porosity and amorphisation occur simultaneously, however, the absence of typical high aspect ratio damage structures and the continuing presence of a crystalline phase clearly differentiates the two cases.

In conclusion, porous structures can be induced in $\mathrm{GaSb}$ by swift heavy ion irradiation, caused by electronic stopping processes. The porous structures differ in comparison to the fibrous networks observed to result from low-energy irradiations. While the results suggest that significant disorder/ amorphisation is required before macroscopic porosity appears, some crystallinity in the form of nanorystallites persists. Lower ion fluences are required in the high-energy case in order to obtain a similar degree of porosity as compared to the low-energy irradiation. This was already apparent in Fig. 1 where the surface region dominated by electronic energy loss has larger void structures than regions where nuclear-stopping dominates, i.e., at the end of range. The significantly different porous morphologies, depending on the energy regime, clearly extend the range of possible applications for the porous structures.

The authors thank the Australian Research Council for support and the staff at the ANU Heavy Ion Accelerator Facility for their continued technical assistance. Part of this research was performed at the SAXS/WAXS beamline at the
Australian Synchrotron. R.C.E. acknowledges the support from the Office of Basic Energy Sciences of the U.S. DOE (Grant No. DE-FG02-97ER45656).

${ }^{1}$ P. Dutta, H. Bhat, and V. Kumar, J. Appl. Phys. 81, 5821 (1997).

${ }^{2}$ A. W. Bett, F. Dimroth, G. Stollwerck, and O. V. Sulima, Appl. Phys. A 69, 119 (1999).

${ }^{3}$ D. Martin, C. Algora, V. Corregidor, and A. Datas, J. Sol. Energy Eng. 129, 283 (2007).

${ }^{4}$ L. Stalmans, J. Poortmans, H. Bender, M. Caymax, K. Said, E. Vazsonyi,

J. Nijs, and R. Mertens, Prog. Photovoltaics 6, 233 (1998).

${ }^{5}$ H. Föll, J. Carstensen, and S. Frey, J. Nanomater. 2006, 91635.

${ }^{6}$ Porous Silicon edited by Z. C. Feng and R. Tsu (World Scientific Publishing Company, Singapore, 1994).

${ }^{7}$ H. Föll, S. Langa, J. Carstensen, M. Christophersen, and I. M. Tiginyanu, Adv. Mater. 15, 183 (2003).

${ }^{8}$ L. M. Wang and R. C. Birtcher, Philos. Mag. 64, 1209 (1991).

${ }^{9}$ R. Callec, P. Favennec, M. Salvi, H. L. Haridon, and M. Gauneau, Appl. Phys. Lett. 59, 1872 (1991).

${ }^{10}$ S. M. Kluth, J. Gerald, and M. C. Ridgway, Appl. Phys. Lett. 86, 131920 (2005).

${ }^{11}$ P. Kluth, S. M. Kluth, B. Johannessen, C. Glover, G. J. Foran, and M. C. Ridgway, J. Appl. Phys. 110, 113528 (2011).

${ }^{12}$ I. H. Wilson, J. Appl. Phys. 53, 1698 (1982).

${ }^{13}$ A. G. Perez-Bergquist, K. Sun, L. Wang, and Y. Zhang, J. Mater. Res. 24, 2286 (2009).

${ }^{14}$ A. Perez-Bergquist, S. Zhu, K. Sun, X. Xiang, Y. Zhang, and L. Wang, Small 4, 1119 (2008).

${ }^{15}$ N. Nitta, M. Taniwaki, Y. Hayashi, and T. Yoshiie, J. Appl. Phys. 92, 1799 (2002).

${ }^{16}$ T. Steinbach, J. Wernecke, P. Kluth, M. C. Ridgway, and W. Wesch, Phys. Rev. B 84, 104108 (2011).

${ }^{17}$ W. Wesch, C. S. Schnohr, P. Kluth, Z. S. Hussain, L. L. Araujo, R. Giulian, D. J. Sprouster, A. P. Byrne, and M. C. Ridgway, J. Phys. D: Appl. Phys 42, 115402 (2009).

${ }^{18}$ T. Steinbach, C. S. Schnohr, P. Kluth, R. Giulian, L. L. Araujo, D. J. Sprouster, M. C. Ridgway, and W. Wesch, Phys. Rev. B 83, 054113 (2011).

${ }^{19}$ J. P. Sullivan, J. Roberts, R. W. Weed, M. R. Went, D. S. Newman, and S. J. Buckman, Meas. Sci. Technol. 21, 085702 (2010).

${ }^{20}$ J. F. Ziegler, J. P. Biersack, and U. Littmark, The Stopping and Range of Ions in Matter (Pergamon, New York, NY, 1985).

${ }^{21}$ G. Szenes, Z. E. Horvath, B. Pecz, F. Paszti, and L. Toth, Phys. Rev. B 65, 045206 (2002).

${ }^{22}$ P. Kluth, C. S. Schnohr, D. J. Sprouster, A. Byrne, D. J. Cookson, and M. C. Ridgway, Nucl. Instrum. Methods Phys. Res. B 266, 2994 (2008).

${ }^{23}$ M. J. Puska and C. Corbel, Phys. Rev. B 38, 9874 (1988).

${ }^{24}$ M. Saito and A. Oshiyama, Phys. Rev. B 53, 7810 (1996).

${ }^{25}$ M. C. Ridgway, T. Bierschenk, R. Giulian, B. Afra, M. D. Rodriguez, L. L. Araujo, A. P. Byrne, N. Kirby, O. H. Pakarinen, F. Djurabekova, K. Nordlund, M. Schleberger, O. Osmani, N. Medvedev, B. Rethfeld, and P. Kluth, Phys. Rev. Lett. 110, 245502 (2013).

${ }^{26}$ P. Kluth, C. S. Schnohr, O. H. Pakarinen, F. Djurabekova, D. J. Sprouster, R. Giulian, M. C. Ridgway, A. Byrne, C. Trautmann, D. J. Cookson, K. Nordlund, and M. Toulemonde, Phys. Rev. Lett. 101, 175503 (2008).

${ }^{27}$ B. Afra, M. Lang, M. Rodriguez, J. Zhang, R. Giulian, N. Kirby, R. C. Ewing, C. Trautmann, M. Toulemonde, and P. Kluth, Phys. Rev. B 83, 064116 (2011).

${ }^{28}$ C. S. Schnohr, P. Kluth, R. Giulian, D. J. Llewellyn, D. J. Cookson, and M. C. Ridgway, Phys. Rev. B 81, 075201 (2010). 